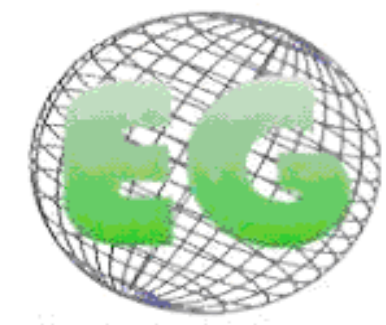

ISSN 1695-6141

$\mathrm{N}^{\circ} 26$
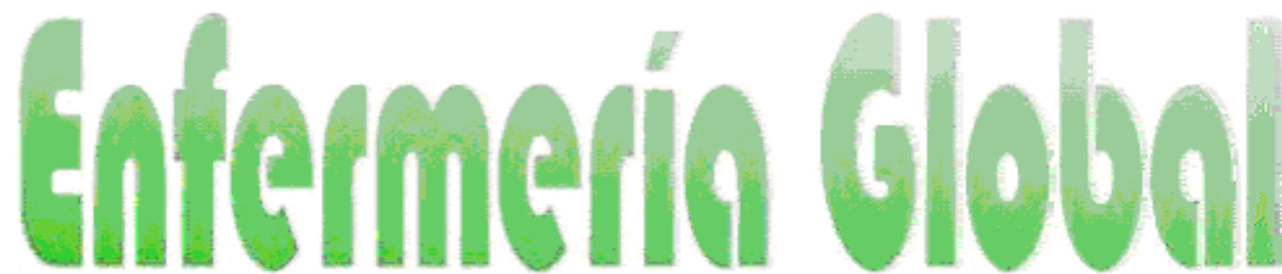

Revista electrónica trimestral de Enfermería

Abril 2012

www.um.es/egloball

\title{
CLINICA
}

\section{Administración de medicamentos: conocimiento de los enfermeros del sector de urgencia y emergencia}

Administração de medicamentos: conhecimento de enfermeiros do setor de urgência e emergencia

\author{
*Machado de Azevedo Filho, F., ${ }^{* *}$ Soares Martins, IM., "**Rodrigues Silva Soares, \\ CS., ${ }^{* *}$ Gomes Fazendeiro, P., ${ }^{* * *}$ Tanferri de Brito Paranaguá, T., ${ }^{* * *}$ Queiroz \\ Bezerra, AL.
}

\begin{abstract}
*Enfermero Intensivista, y docente em la Facultad Estácio de Sá de Goiás. E-mail francino21@gmail.com
**Estudiante de $8^{\circ}$ período del curso de graduación en enfermería de la Facultad Estácio de Sá de Goiás.*** Enfermera. Doctoranda del Programa de Pos-Graduación en Enfermería de la Facultad de Enfermería de la Universidad Federal de Goiás. ****Doctora. Enfermera. Profesora Asociadao de la Facultad de Enfermería de la Universidad Federal de Goiás.Brasil.
\end{abstract}

Palabras clave: enfermería; unidad de urgencia/emergencia; administración de medicamentos; conocimiento. Palavras chaves: enfermagem; unidade de urgência/emergência; administração de medicamentos; conhecimento.

Keywords: Nursing; emergency and urgency units; drugs administration; knowledge

\section{RESUMEN}

La intervención medicamentosa es una aliada en el proceso de recuperación de la salud, principalmente en el sector de urgencia y emergencia. Al ser el enfermero el principal responsable de esa práctica y considerando que cualquier fallo durante esta actividad puede tener consecuencias irreversibles para el paciente, el estudio objetiva describir el conocimiento de los enfermeros acerca de esta temática y verificar los aspectos de la formación, en busca de actualización y educación continuada en el servicio de salud.

Estudio descriptivo, exploratorio, de abordaje cuantitativo, realizado en unidades de urgencia y emergencia del Distrito Sanitario Leste de Goiânia, Goiás.

Participaron del estudio 37 enfermeros que respondieron a un cuestionario auto-aplicable. Se realizó análisis estadístico y descriptivo, considerando intervalo de $95 \%$ de confianza y factores con $p<0,05$ significantes.

En el estudio, se constata la preparación de los enfermeros en cuanto a la administración de medicamentos en urgencia y emergencia y apunta lagunas que deben ser subsanadas por medio de la educación continuada. 


\section{RESUMO}

A intervenção medicamentosa é uma aliada no processo de recuperação da saúde, principalmente no setor de urgência e emergência. Tendo o enfermeiro como o principal responsável por essa prática e considerando que qualquer falha durante esta atividade pode trazer conseqüências irreversíveis ao paciente, o estudo objetiva descrever o conhecimento dos enfermeiros acerca desta temética e verificar os aspectos da formação, busca de atualização e educação continuada no serviço de saúde.

Estudo descritivo, exploratório, de abordagem quantitativa, realizado em unidades de urgência e emergência do Distrito Sanitário Leste de Goiânia, Goiás.

Participaram do estudo 37 enfermeiros que responderam um questionário auto-aplicável. Realizou-se análise estatística e descritiva, considerando intervalo de $95 \%$ de confiança e fatores com $p<0,05$ significantes.

Pelo estudo, constata-se o preparo dos enfermeiros quanto à administração de medicamentos em urgência e emergência e aponta lacunas que devem ser sanadas por meio da educação continuada.

\section{ABSTRACT}

Administration of medicines is an ally in the health recovery process, especially in the emergency and casualty sector. Since the nurse is the person with most responsibility in this practice and considering that any failing in this activity could have irreversible consequences for the patient, the study seeks to describe the knowledge of the nurses about this issue and to verify aspects of their training in a search for continuous updating and education in the health service.

The study is descriptive and exploratory and of a quantitative approach. It was carried out in casualty and emergency units of the Distrito Sanitario Leste de Goiânia, Goiás.

37 nurses participated in the study, answering a self-administered questionnaire. A statistical and descriptive analysis was carried out, taking a confidence interval of $95 \%$ and factors with significance $p<0,05$.

The study shows the training of nurses in terms of medicine administration in casualty and emergency units and highlights gaps that need to be remedied by ongoing training.

\section{INTRODUCCIÓN}

El sector de urgencias y emergencias es un área de alto riesgo para la ocurrencia de eventos indeseables, debido a la rotatividad y dinámica de atención, por la gran demanda de pacientes con varios grados de gravedad, por la deficiencia cuantitativa y cualitativa de los recursos humanos y materiales, sobrecarga de trabajo y estrés profesional y ambiental ${ }^{(1,2)}$.

Frente a las características clínicas de los pacientes atendidos en la unidad de urgencia y emergencia, la intervención medicamentosa y administración de drogas con alto poder de acción es frecuente convirtiéndose en una aliada en el proceso de recuperación de la salud. Por ello, la administración de medicamentos exige un cuidado intenso y requiere conocimientos específicos y especializados, pues cualquier fallo durante esta actividad puede acarrear consecuencias tales como reacciones adversas, reacciones alérgicas y errores de medicación, los cuales pueden ser irreversibles y devastadores ${ }^{(1,3,4)}$.

Recientes estudios evidencian que los errores en la administración de medicamentos representan una triste realidad del servicio de salud, repercutiendo negativamente en los indicadores de asistencia y en los resultados institucionales ${ }^{(3)}$.

En Brasil, el enfermero es el profesional responsable del proceso de administración de medicamentos, constituyéndose en líder del equipo de enfermería y asumiendo un papel fundamental tanto en el cuidado al paciente que se encuentra en terapia medicamentosa como en la propagación del conocimiento acerca de esta práctica para el equipo. Entre los 
cuidados en el proceso de administración de medicamentos destacan la evaluación de la pre-administración y dosis, vigilancia de los efectos terapéuticos, identificación y reducción de efectos adversos, prevención de interacciones medicamentosas y control de la toxicidad $^{(4,5)}$.

Por el hecho de que la enfermería actúa esencialmente en el final del proceso de la terapia medicamentosa, aumenta su responsabilidad en evidenciar e impedir fallos, pues la acción de administrar es la última oportunidad de interrumpir el sistema, evitando errores que por suerte sucedieron en las primeras fases de este proceso, tales como prescripción y cálculo de dosis ${ }^{(6)}$.

De esta forma, ante la gravedad y complejidad de las ocurrencias iatrogénicas con medicación, es necesaria la aplicación de varios principios científicos que fundamenten la acción del enfermero, para prevenir y reducir errores, dar la seguridad necesaria al cliente y garantizar la calidad del servicio. Sin embargo, se verifica lo poco que se sabe acerca de las causas, factores y conductas de los enfermeros ante estas situaciones.

Los cinco ciertos es un modelo para prevención de errores en la administración de medicamentos que, a pesar de ser poco integral y abordar solo aspectos individuales del profesional, ignorando factores relacionados con el sistema de medicación, continúa dominante en la actualidad. Este modelo es válido, pero no es totalmente suficiente para prevenir la ocurrencia de errores en la administración de medicamentos ${ }^{(5)}$.

De esta forma, para seguir el raciocinio lógico de este modelo y garantizar la eficiencia y seguridad del paciente durante la terapia medicamentosa, es imprescindible tener conocimiento lo que se configura como la mejor forma de prevenir la ocurrencia de errores ${ }^{(4)}$.

De acuerdo con el Código de Ética de los Profesionales de Enfermería el enfermero debe prestar cuidados en salud libre de los daños causados por la impericia, negligencia e imprudencia. Para ello, debe buscar fundamento teórico/práctico que ayude sus actividades, mejorar sus conocimientos técnicos, científicos, éticos y culturales, en beneficio de la persona, familia y colectividad y del desarrollo de la profesión, estando prohibido administrar medicamentos sin conocer la acción de la droga y sin asegurarse de los riesgos ${ }^{(7)}$.

Así, la formación continuada es una herramienta que permite el desarrollo de los profesionales de salud y asegura la calidad de la atención a los clientes. Para la eficacia de la formación continuada en el servicio, el gestor debe considerar la realidad institucional y actuar directamente sobre las necesidades del profesional, instigando el real interés del equipo ante las situaciones cotidianas ${ }^{(8)}$.

Es a través del conocimiento como se alcanzan las competencias para una actuación profesional cualificada, segura y libre de riesgos. Ante lo expuesto, investigar el conocimiento de los profesionales acerca de la administración de medicamentos en urgencia y emergencia ayudará al plan de estrategias educativas, así como apoyará programas de educación continuada, contribuyendo al desarrollo de enfermeros y mejora del desempeño de los servicios de salud.

\section{OBJETIVO}

Describir el conocimiento de los enfermeros acerca de la administración de medicamentos en urgencia y emergencia, así como verificar los aspectos de la formación, busca de actualización y formación continuada en el servicio de salud. 


\section{METODOLOGÍA}

Se trata de un estudio descriptivo, exploratorio, de enfoque cuantitativo, desarrollado en tres unidades de urgencia y emergencia del Distrito Sanitario Leste de Goiânia, Goiás, vinculadas a la Secretaría Municipal de Salud.

La muestra del estudio consta de 37 enfermeros actuantes en estas unidades, que atendieron los siguientes criterios de inclusión: estar en activo durante el período de recogida de datos; aceptar participar voluntariamente del estudio suscribiendo el Término de Consentimiento Libre y Esclarecido; responder completamente al cuestionario de recogida de datos.

La recogida de datos se realizó en el mes de octubre de 2010, utilizando un cuestionario auto-aplicable, estructurado y validado, dividido en tres partes pre-categorizadas. La parte I aportó datos sobre caracterización de los sujetos, aspectos de la formación, percepción del conocimiento en administración de medicamentos y busca de actualización; la parte II investigó el enfoque de la administración de medicamentos en urgencia y emergencia en cuanto formación continuada en el servicio de salud; y la parte III constituye un test de conocimientos teniendo como variables de análisis cuestiones sobre administración de medicamentos en urgencia y emergencia retiradas de compilaciones de concursos en el área de enfermería y pre-tests específicos.

Los datos obtenidos fueron digitados, con doble conferencia, en el banco de datos del programa SPSS (Statistical Package For The Social Science versão 11,5 para Windows). Se realizó análisis estadístico y descriptivo de las variables, considerando intervalo de $95 \%$ de confianza y factores con $p<0,05$ significantes. Los resultados se presentaron en forma de frecuencia absoluta y relativa, tablas y gráficos y discutidos con base en literatura científica.

Estudio apreciado por el Comité de Ética en Investigación del Hospital de Urgencia de Goiânia, Goiás, recibiendo parecer favorable bajo Protocolo №058/10. Todos los sujetos de la investigación recibieron y suscribieron el Término de Consentimiento Libre y Esclarecido, atendiendo todas las directrices y normas reglamentadas por la Resolución 196/96 ${ }^{(9)}$.

\section{RESULTADOS Y DISCUSIÓN}

El Distrito Leste Sanitário tiene 46 enfermeros distribuidos en las tres unidades de urgencia y emergencia campos de este estudio. Durante el período de recogida de datos, dos estaban de vacaciones, cinco de licencia, uno renunció a participar del estudio y fue excluido un cuestionario por no contener todas las respuestas imprescindibles para el alcance de los objetivos, participando 37 enfermeros.

Para una mejor discusión, presentaremos los resultados conforme a la división del cuestionario.

\section{Caracterización de los Enfermeros}

Los datos sobre la caracterización de los enfermeros están presentados en la Tabla 1. 
Tabla 1 - Distribución de los enfermeros actuantes en las unidades de urgencia y emergencia del Distrito Sanitario Leste de Goiânia, Goiás, 2010.

\begin{tabular}{|c|c|c|}
\hline \multicolumn{3}{|c|}{$\begin{array}{l}\text { Caracterización de los enfermeros actuantes en } \\
\text { las unidades de urgencia y emergencia del } \\
\text { Distrito Sanitario Leste de Goiânia, Goiás, } 2010 \text {. }\end{array}$} \\
\hline & $\mathrm{n}$ & $f(\%)$ \\
\hline \multicolumn{3}{|l|}{ Sexo } \\
\hline Femenino & 34 & 91,9 \\
\hline Masculino & 3 & 8,1 \\
\hline \multicolumn{3}{|l|}{ Edad (en años) } \\
\hline $22-28$ & 15 & 40,5 \\
\hline $30-36$ & 14 & 37,9 \\
\hline $37-44$ & 4 & 10,8 \\
\hline 46 o más & 4 & 10,8 \\
\hline \multicolumn{3}{|l|}{ Estado civil } \\
\hline Soltero & 17 & 45,9 \\
\hline Casado & 18 & 48,6 \\
\hline Divorciado & 2 & 5,4 \\
\hline \multicolumn{3}{|l|}{ Turno de trabajo } \\
\hline Matutino & 2 & 5,4 \\
\hline Vespertino & 3 & 8,1 \\
\hline Nocturno & 10 & 27,0 \\
\hline Diurno & 21 & 56,8 \\
\hline Alternativo & 1 & 2,7 \\
\hline \multicolumn{3}{|l|}{ Vínculo de trabajo } \\
\hline Uno & 21 & 56,7 \\
\hline Dos & 12 & 32,4 \\
\hline Tres o más & 1 & 2,7 \\
\hline No responden & 3 & 8,1 \\
\hline \multicolumn{3}{|l|}{ Especialización } \\
\hline Sí & 23 & 62,2 \\
\hline No & 14 & 37,8 \\
\hline \multicolumn{3}{|l|}{ Titulación } \\
\hline Licenciado & 14 & 37,8 \\
\hline Especialista & 23 & 62,2 \\
\hline Total & 37 & $100 \%$ \\
\hline
\end{tabular}

La mayoría de los enfermeros es del sexo femenino, lo que no contradice el contexto de la enfermería. Estudios que estudian la distribución de los profesionales de enfermería en hospitales brasileños muestran que el trabajo es ejercido casi exclusivamente por mujeres $^{(10)}$, lo que puede ser explicado por la propia retrospectiva histórica de la profesión. 
La media de edad fue de 32,08 años, con mínima de 22 y máxima de 56, siendo la franja de edad predominante de 22 a 28 años. En cuanto al estado civil, predominaron los casados y solteros.

La guardia diurna de 12 horas fue el turno de trabajo más referido, seguido del nocturno. Pocos relataron trabajar solo en un periodo. Otro horario de trabajo practicado por los enfermeros, denominado alternativo, comprende un horario intermedio, organizado para facilitar el servicio y no perjudicar la continuidad de la asistencia durante los cambios de guardia. Todos los participantes cumplen carga horaria de 30 horas semanales.

Más de la mitad de los enfermeros trabajan en régimen de dedicación exclusiva. Sin embargo, 35,1\% de ellos dijeron tener dos o más vínculos. El trabajo con carácter de dedicación exclusiva es un factor positivo dado que posibilita la mejora profesional y formación de vínculo tanto organizacional como con el usuario. Investigadores afirman que la precarización de los salarios obliga a los profesionales a tener más de un vínculo de trabajo, resultando una carga mensual larga, exhaustiva y desgastante ${ }^{(10,11)}$, lo que desmotiva a los profesionales a buscar nuevos conocimientos.

Por ello es necesario estimular el carácter de dedicación exclusiva, a fin de evitar sobrecarga de trabajo, déficit de atención, fatiga, falta de tiempo para dedicarse a la planificación de la asistencia, así como para mantenerse actualizado.

La Especialización fue la mayor titulación referida siendo que siete enfermeros $(18,9 \%)$ son especialistas en UTI, seis (16.2\%) en Salud Pública, tres $(8,1 \%)$ en Estrategia Salud de Familia y dos $(5,4 \%)$ en Auditoría del Sistema de Salud. Solo tres $(8,1 \%)$ refirieron especialización en Urgencia y Emergencia. Otros cursos citados fueron Obstetricia, Neopediatría, Centro Quirúrgico/Centro de Material y Esterilización y Gestión de Sistema de Servicios de Salud.

La especialización posibilita al profesional de salud mantenerse en constante competitividad en el mercado de trabajo, además de configurarse en busca del saber científico que le garantice seguridad en el servicio asistencial y primordial para la emancipación del conocimiento técnico, descubriéndoles un nuevo aprender ${ }^{(12)}$.

Teniendo en cuenta que el contexto de la salud se ha desarrollado mucho en los últimos años en cuanto a investigación y tecnología, es necesario estimular a los enfermeros a acompañar este avance, apuntando un cambio de actitudes y comportamientos.

El tiempo de formación varió de 10 meses a 25 años y seis meses, mientras que el tiempo de actuación en emergencia y urgencia fue de ocho meses a 18 años, con media de tres años y franja predominante de ocho meses a dos años para 23 enfermeros $(62,2 \%)$.

Al ser preguntados sobre el nivel de conocimiento en administración de medicamentos en urgencia y emergencia, 28 (75,7\%) refirieron ser bueno y los demás respondieron ser malo.

En cuanto a la participación en cursos de actualización en administración de medicamentos, se observó que solo seis enfermeros $(16,2 \%)$ refirieron tener realizado curso de corta duración, tres $(8,1 \%)$ refirieron no recordarlo y $28(75,7 \%)$ afirmaron la no realización.

La comparación entre la percepción en cuanto al nivel de conocimientos en administración de medicamentos y el tiempo de servicio en urgencia y emergencia indicó que los enfermeros que consideran tener un buen nivel de conocimiento poseen de ocho meses a 
16 años de actuación en urgencia y emergencia, con media de tres años y seis meses. En cambio los enfermeros que consideran su nivel de conocimiento malo, poseen de 10 meses a cuatro años de experiencia, con media de un año y nueve meses, evidenciando que el mayor tiempo de actuación en urgencia y emergencia da al profesional la percepción de una mejor preparación y conocimiento sobre la administración de medicamentos.

Sin embargo, el cruce de las variables percepción del nivel de conocimiento y realización de cursos de actualización en administración de medicamentos, Cuadro 1, todos los que afirmaron haber realizado curso refirieron tener buen conocimiento.

Cuadro 1 - Distribución de las variables 'nivel de conocimiento en administración de medicamentos en urgencia y emergencia' y 'realización de cursos' sobre el tema. Goiânia, Goiás, 2010.

\begin{tabular}{|c|l|l|l|l|}
\hline \multirow{2}{*}{$\begin{array}{l}\text { NIVEL DE } \\
\text { CONOCIMIENTO }\end{array}$} & \multicolumn{3}{|c|}{ REALIZÓ CURSO } & Total \\
\cline { 2 - 5 } & Sí & NO & $\begin{array}{l}\text { NO SE } \\
\text { ACUERDA }\end{array}$ & \\
\hline BUENO & 6 & 21 & 1 & 28 \\
Total & 0 & 7 & 2 & 9 \\
\hline
\end{tabular}

Estudios publicados en los últimos años sobre conocimientos de los enfermeros en relación al uso de medicamentos específicos identificaron que $79,2 \%$ de los enfermeros entrevistados informaron que el conocimiento de farmacología adquirido durante la graduación fue insuficiente para la práctica profesional, $81,1 \%$ consideraron que el contenido dado y la carga horaria de la disciplina fueron insuficientes y $96,2 \%$ informaron que la relación de la teoría de farmacología con la práctica fue insatisfactoria ${ }^{(5)}$.

De ahí la importancia de la constante busca de actualización, a fin de posibilitar el cubrir las lagunas que el curso de graduación no alcanzó, ya que para un tratamiento eficaz durante la terapia medicamentosa, es fundamental que el profesional domine el conocimiento técnico y científico $^{(13)}$.

Estudio realizado en un hospital en el interior de São Paulo demostró que los enfermeros son frecuentemente interrogados por técnicos y auxiliares de enfermería, colegas de profesión y médicos, en busca de informaciones, para suplir dudas sobre medicaciones, principalmente en lo referente a disolución, administración, infusión, preparación e indicaciones del medicamento ${ }^{(4)}$.

Ante lo expuesto, es evidente la importancia de incentivar y estimular la realización de cursos por la enfermería especialmente en el área de administración en urgencia y emergencia, pues la falta de conocimiento sobre esta temática puede perjudicar la asistencia en varios aspectos.

Es necesaria, también, la utilización de métodos como formación continuada, atendiendo al crecimiento intelectual y práctico de los profesionales de enfermería ${ }^{(13)}$. 


\section{Administración de medicamentos en cuanto a formación continuada en el servicio}

La formación continuada tiene el objetivo de preparar al profesional de salud para una actuación con mejor calidad de servicio.

En cuanto a un programa sistematizado de formación continuada que contemple la temática administración de medicamentos en urgencia y emergencia, todos los enfermeros fueron unánimes en afirmar su no existencia en el servicio. Por otro lado, $22(59,4 \%)$ enfermeros refirieron que esa temática nunca fue abordada por el servicio, $11(29,7 \%)$ afirmaron que el tema ya fue abordado en otros momentos, tres $(8,1 \%)$ refirieron ser frecuente y uno $(2,7 \%)$ no respondió.

La divergencia de respuestas infiere que el enfoque administración de medicamentos es realizado, mas no integra a todos los profesionales vinculados al sector, lo que puede ser explicado por los diferentes turnos de trabajo.

Es esencial el continuo perfeccionamiento de los trabajadores de la salud, siendo la formación continuada de gran importancia dentro de las instituciones, donde la temática se contemple y sea comprendida por todo el equipo de salud ${ }^{(8)}$, evidenciando la necesidad de mejorar la planificación de las actividades de formación continuada desarrollada por los servicios.

Se refuerza también la importancia del predominio de acciones educativas pautadas en conocimiento técnico-científico con énfasis en cursos y prácticas orientadas ${ }^{(14)}$.

Al ser preguntados sobre si habían recibido prácticas sobre el proceso de administración de medicamentos en el acto de admisión en la unidad de emergencia, solamente tres $(8,1 \%)$ refirieron haberlas recibido. Sin embargo, todos reconocen la importancia de recibir cursos, para la prevención de errores, por ser esta la principal categoría responsable de la administración de medicamentos y por contribuir a la práctica profesional.

Todos los días nuevas drogas son introducidas en el mercado trazando otras posibilidades para tratamiento, reforzando la necesidad de actualización continua ${ }^{(15)}$. Así, es importante la existencia de prácticas, cursos como continuación o extensión del modelo académico ${ }^{(14)}$, a fin de mantener una cualificación adecuada con el servicio, así como evidenciar fallos en habilidades que exigen práctica y desarrollar competencias profesionales.

En cuanto a la existencia de protocolos, manuales y/o guías de preparación, disolución y administración de medicamentos, 26 (70,3\%) respondieron que no hay ninguno de esos documentos en el sector, seis $(16,2 \%)$ respondieron que sí, cuatro $(10,8 \%)$ afirmaron no saber y uno $(2,7 \%)$ no respondió.

Estudios señalan que la utilización de protocolos genera un aumento en la tasa de supervivencia del $80 \%$, representando un progreso en el desarrollo de nuevos medicamentos y protocolos terapéuticos ${ }^{(16)}$.

De esta forma, el uso de protocolos, manuales y/o guías en la asistencia es de extrema importancia para el servicio, pues ayuda a las acciones desarrolladas por los profesionales de salud, permite la mejora de la calidad de la asistencia, da mayor seguridad al profesional en el acto de cualquier procedimiento / intervención, así como garantiza la seguridad del paciente. 


\section{Conocimientos básicos sobre administración de medicamentos en urgencia y emergencia}

El número total de cuestiones del test de conocimientos básicos sobre administración de medicamentos en unidades de urgencia y emergencia fue nueve y los aciertos variaron de tres a ocho, conforme distribución del Gráfico 1.

Gráfico 1 - Distribución de los enfermeros conforme número de aciertos en el test de farmacología básica en urgencia/emergencia. Goiânia, Goiás, 2010.

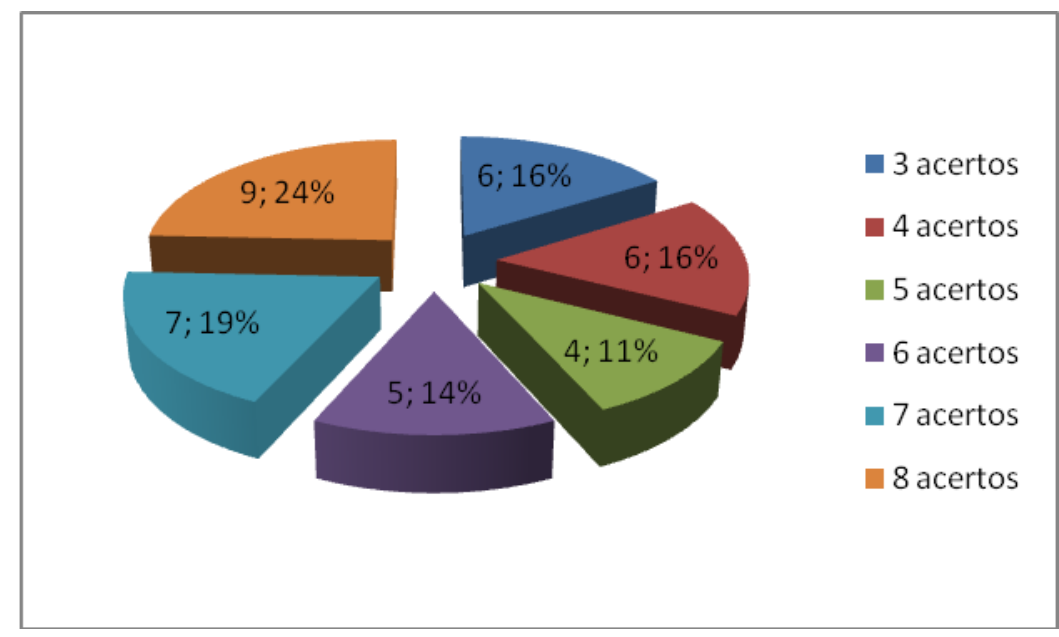

Doce $(32,4 \%)$ enfermeros acertaron menos de la mitad y el mayor rendimiento fue igual a $88,8 \%$, con ocho aciertos, para $24,3 \%$ de los enfermeros.

Estudio semejante indicó que, tras la aplicación de un test sobre conocimientos de los enfermeros en relación al uso de medicamentos específicos, 41,5\% acertaron menos de la mitad de las cuestiones propuestas sobre la utilización de los fármacos. Estos datos indican una posible falta de preparación de los enfermeros para supervisar la administración de medicamentos ya que esto exige de ellos un buen conocimiento de farmacología ${ }^{(5)}$.

En este sentido, la falta de conocimiento sobre administración de medicamentos en urgencia y emergencia genera preocupaciones, pues puede llevar a la ocurrencia de errores, generando riesgos al paciente, profesional e institución ${ }^{(3)}$.

La relación entre el mayor o menor tiempo de experiencia en urgencia y emergencia y el número de aciertos en el test no apuntó significancia. Sin embargo, del cruce de las variables número de aciertos y realización de cursos de actualización en administración de medicamentos, se obtuvo una media de 6,5 aciertos para aquellos que realizaron, con mediana igual a siete; media de 5,67 y mediana igual a seis puntos para los que refirieron no acordarse y media de 5,61 puntos y mediana de 5,5 para aquellos que no realizaron cursos de actualización.

Aunque la diferencia de aciertos en el test aplicado a los enfermeros no sea alarmante, el resultado evidencia un mejor aprovechamiento de aquellos que realizaron cursos de actualización, corroborando con investigaciones que defienden la importancia de la busca de la cualificación profesional y formación continuada ${ }^{(8,14,15)}$. 
El Gráfico 2 presenta la distribución de los enfermeros en cuanto al número de aciertos y de errores.

Gráfico 2 - Distribución de las cuestiones en cuanto a número de errores, aciertos y no respondidas. Goiânia, Goiás, 2010.

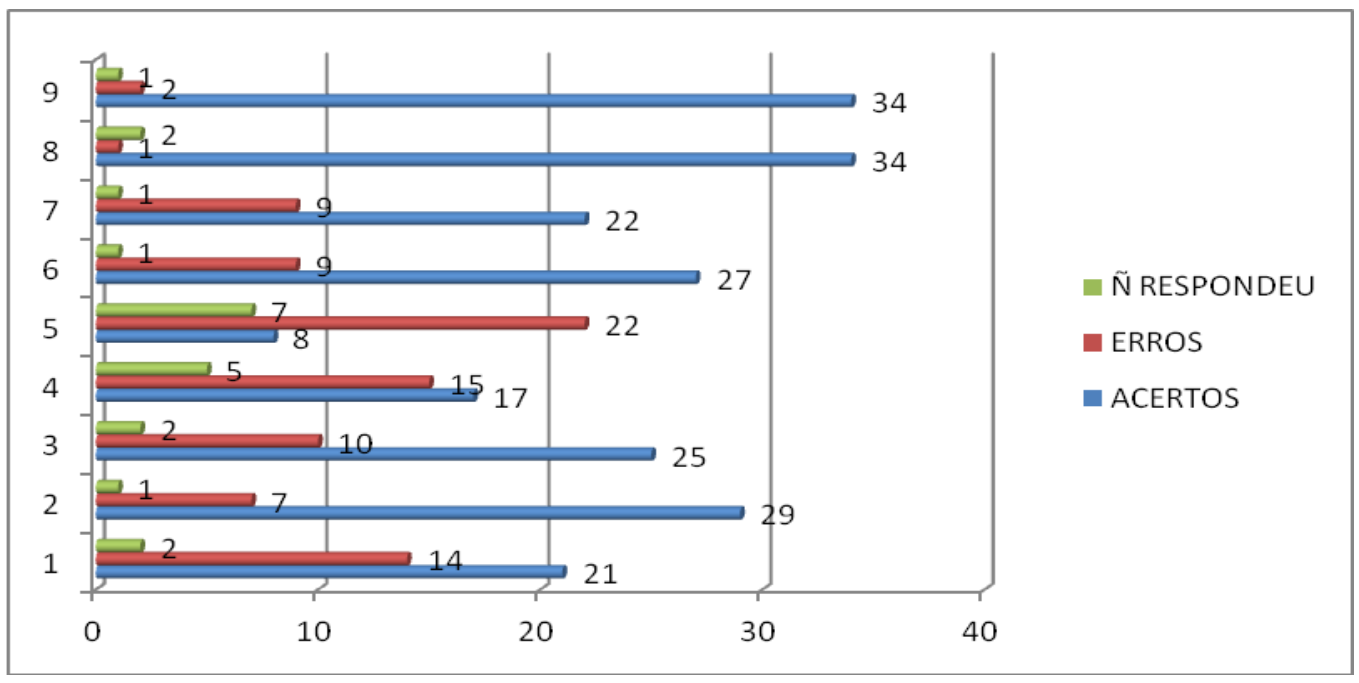

Las cuestiones con menor aprovechamiento fueron, respectivamente, 6, 5, 1 y 8.

La pregunta 6 investiga el conocimiento sobre el ritmo cardiaco considerado letal ante la aplicación de lidocaína, la cual obtuvo 59,5\% de errores, mostrando la necesidad del desarrollo de acciones educativas que aclaren dudas sobre la temática.

La lidocaína tiene como efecto hipotensión y bradicardia debido a la reacción del agente analgésico en los componentes del sistema cardiovascular y/o en los receptores betaadrenérgicos estimulando la acción de la epinefrina ${ }^{(17)}$. En el ritmo idioventricular la frecuencia cardiaca cae a menos de 40bpm antecedido de ritmos más altos que fueron inhibidos $^{(18)}$. Sin embargo, la lidocaína, si se administra en pacientes con ritmo idioventricular, acentúa la desaceleración del ritmo cardiaco pudiendo así causar una parada cardiaca.

La cuestión 5 obtuvo 40,5\% de errores y está relacionada con los inhibidores de la enzima conversora de la angiotensina (ECA). Los inhibidores de la ECA son eficaces en el control anti-hipertensivo y conocer su acción es extremadamente importante por permitir la disminución de eventos cardiovasculares en pacientes de alto riesgo ${ }^{(19)}$.

Las preguntas 1 y 8 tuvieron $37,8 \%$ de errores y se refieren, respectivamente a la administración de drogas por vía endotraqueal y el intervalo ideal de las dosis de atropina en una parada cardiopulmonar.

Es importante que el enfermero sepa las opciones de las vías de administración de los medicamentos usados en la urgencia y emergencia, pues su desconocimiento es una de las principales causas de errores. Cuando el acceso intravenoso o intraóseo está imposibilitado o, también, cuando precisamos de otra vía de mejor absorción, se opta por la administración endotraqueal. La naloxona, atropina, epinefrina y lidocaína, son drogas que pueden ser administradas por la vía endotraqueal ${ }^{(20)}$. 
En cuanto al intervalo ideal de las dosis de atropina, la literatura apunta un intervalo de 3 a 5 minutos, a fin de controlar la asistolia con ritmos bradicárdicos ${ }^{(21)}$.

Las preguntas 9, 10, 2, 7 y 3, tuvieron, en este orden, mejor aprovechamiento, variando de $67,6 \%$ a $91,9 \%$ de aciertos.

Las preguntas 9 y 10 tuvieron aprovechamiento de $91,9 \%$ y se referían, respectivamente, a los signos que deben ser monitorizados en el paciente que recibe dosis de morfina y sobre la medicación indicada para cesar un cuadro de crisis convulsiva prolongada.

Los efectos más serios observados durante la administración de la morfina son: depresión respiratoria, hipotensión arterial, taquicardia y otras manifestaciones del sistema nervioso central, tales como euforia, malestar, debilidad, dolor de cabeza, insomnio, agitación, desorientación, problemas visuales y vértigo ${ }^{(17)}$. Así, los principales cuidados frente a la administración de la morfina son la monitorización de la frecuencia respiratoria, presión arterial, pulso y nivel de conciencia.

En cuanto al tratamiento de la crisis convulsiva prolongada, se recomienda la administración de Diazepan. El diazepan está indicado en el tratamiento de estados de excitación asociados a la ansiedad aguda y pánico, así como en la agitación motora, espasmo muscular reflejo y tratamiento agudo del status epilepticus y otros estados convulsivos ${ }^{(17)}$.

La pregunta 2, con $78,4 \%$ de aciertos, investigaba los efectos colaterales de la amiodarona. Los efectos más comunes en la administración de la amiodarona son bradicardia, generalmente moderada y dosis dependiente e hipotensión, la cual puede ser evitada con una administración lenta ${ }^{(17)}$.

La pregunta 7 se refiere a la finalidad de la utilización del bicarbonato de sodio en una parada cardiaca (PCR) y tuvo $72,9 \%$ de aciertos. El bicarbonato de sodio es utilizado para el control de la acidosis metabólica en las fases tardías de la PCR (>10 min) o en los casos en que se sospeche de acidosis previa ${ }^{(21)}$.

La pregunta 3 abordaba el mecanismo de acción de la epinefrina y vasopressina en una parada respiratoria y tuvo aprovechamiento de $67,6 \%$. La vasopressina es una hormona con efecto antidiurético y vasoconstructor. También posee acción hemostática, efectos en la termorregulación y es un segregador de la hormaona adrenocorticotrópica ${ }^{(22)}$.

A partir del test de conocimientos básicos sobre la utilización de medicamentos en urgencia y emergencia, se observa la preparación de los enfermeros en cuanto a la temática y apunta lagunas que deben ser subsanadas por medo de la formación continuada.

\section{CONSIDERACIONES}

En el estudio se verificó que la mayoría de los enfermeros es de sexo femenino, casado, con media de edad igual a 32,08 años, variando de 22 a 56 años. El turno de trabajo predominante es el diurno y más de la mitad de los enfermeros trabajan en régimen de dedicación exclusiva.

La titulación de especialista fue la mayor encontrada, siendo que una parcela mínima refirió especialización en Urgencia y Emergencia, evidenciando la necesidad de estimular a los enfermeros para la busca continua de mejor formación y cualificación para el trabajo en urgencia y emergencia. 
Los enfermeros con mayor tiempo de experiencia en este sector consideran tener un buen conocimiento en farmacología, al contrario de aquellos con menor tiempo. Sin embargo, todos los que afirmaron tener realizado curso de actualización en farmacología refirieron tener buen conocimiento, evidenciando la importancia de actualizarse.

La formación continuada en el servicio es una realidad, mas muestra la necesidad de mejor la sistematización a fin de llegar a todos los profesionales de salud y alcanzar mejor desempeño del servicio.

El test de conocimientos básicos de administración de medicamentos en unidades de urgencia y emergencia demostró rendimiento variable, con mayor rendimiento igual a $88,8 \%$ y demostró que no hay influencia del tiempo de experiencia. Sin embargo, la media de aciertos fue mayor para los enfermeros que afirmaron tener realizados cursos de actualización en farmacología.

A partir del test de conocimientos básicos sobre la utilización de medicamentos en urgencia y emergencia, se observa la preparación de los enfermeros en cuanto a la temática y apunta lagunas que deben ser subsanadas por medio de la formación continuada.

Destaca que esta investigación es un recorte en el contexto en que se inscribe la enfermería, presentando limitaciones en el alcance de sus resultados, mas traza avances sobre el conocimiento de los enfermeros sobre la administración de medicamentos en urgencia y emergencia, práctica frecuente en el cotidiano de los enfermeros.

Esperamos que el estudio contribuya a la planificación de estrategias de desarrollo de personal en enfermería y salud, subsidiando la praxis del cuidado para un mejor desempeño profesional, calidad del servicio y seguridad del paciente.

\section{REFERENCIAS BIBLIOGRÁFICAS}

1.Oliveira RC, Camargo AEB, Cassiani SHB. Estratégias para prevenção de erros na medicação no setor de emergência. Rev Bras Enferm. 2005; 58(4):399-404.

2.Souza RB, Silva MJP, Nori A. Pronto-Socorro: uma visão sobre a interação entre profissionais de enfermagem e pacientes. Rev Gaúcha Enferm. 2007; 28(2):242-9.

3.Silva BK, Silva JS, Gobbo AFF, Miasso Al. Erros de medicação: condutas e propostas de prevenção na perspectiva da equipe de enfermagem. Rev Eletr Enferm [periódico na internet]. 2007 [citado 2010 set. 28]; 9(3):[cerca de 11 p]. Disponível em: http://www.fen.ufg.br/revista/v9/n3/v9n3a11.htm.

4.Silva DO, Grou CR, Miasso AI, Cassiani SHB. Preparo e administração de medicamentos: análise de questionamentos e informações da equipe de enfermagem. Rev Latino-Am Enferm [periódico na internet]. 2007 [citado 2010 set. 28]; 15(5):[cerca de 8 p]. Disponível em: $\quad$ http://www.scielo.br/scielo.php?pid=S010411692007000500020\&script=sci_abstract\&tlng=pt pdf.

5.Santana ARCMBF. Conhecimento de Enfermeiros de Clínica Médica e Unidade de Terapia Intensiva de Hospitais Escola da Região Centro-Oeste sobre medicamentos específicos [dissertação]. Ribeirão Preto (SP): Escola de Enfermagem, Universidade de São Paulo; 2006.

6.Miasso Al, Silva AEBC, Cassiani SHB, Grou CR, Oliveira RC, Fakih FT. O processo de preparo e administração de medicamentos: identificação de problemas para propor melhorias e prevenir erros de medicação. Rev Latino-Am Enferm [periódico na internet]. 
2006 [citado 2010 set. 28]; 14(3):[cerca de 9 p]. Disponível em: http://www.scielo.br/pdf/rlae/v14n3/v14n3a08.pdf.

7.Brasil. Conselho Federal de Enfermagem. Resolução COFEN no 311/2007. [citado 2010 dez 2] Disponível

em:

$<$ www.huwc.ufc.br/arquivos/biblioteca_cientifica/1188236444_91_0.pdf>.

8.Silva MF, Conceição FA, Leite MMJ. Educação continuada: um levantamento de necessidades da equipe de enfermagem. O Mundo da Saúde. 2008; 32(1):47-55.

9.Conselho Nacional de Saúde. Resolução n. 196, de 10 de outubro de 1996. Dispõe sobre diretrizes e normas regulamentadoras de pesquisas envolvendo seres humanos. Bioética. 1996;4(2 Supl):15-25.

10.Branquinho NCSS, Bezerra ANQ, Abraão SR, Paranaguá TTB, Ramalho WS. Processo de seleção de enfermeiros de um hospital de ensino da região Centro Oeste. Rev enferm UERJ. 2010; 18(3):394-9.

11.Nóbrega MFB, Matos MG, Silva LMS, Jorge MSB. Perfil gerencial de enfermeiros que atuam em um hospital público federal de ensino. Rev enferm UERJ. 2008; 16(3):333-8.

12.Oliveira NA, Thofehrn MB, Cecagno D, Siqueira HCH, Porto ARutz. Especialização em projetos assistenciais de enfermagem: contribuições na prática profissional dos egressos. Texto Contexto Enferm. 2009; 18(4):697-704.

13.Silva LD, Henrique DM, Shutz V. Ações do enfermeiro na terapia farmacológica para o acidente vascular cerebral. Rev enferm UERJ. 2009;17(3);423-9.

14.Montanha D, Peduzzi M. Educação permanente em enfermagem: levantamento de necessidades e resultados esperados segundo a concepção dos trabalhadores. Rev Esc Enferm USP. 2010; 44(3):597-604

15.Bortolotto LA, Malachias MVB. Novos medicamentos na hipertensão resistente. Rev Bras Hipertens. 2009; 16(Suppl 1): S13-S15.

16.Cazé MO, Bueno D, Santos MEF. Estudo referencial de um protocolo quimioterápico para leucemia linfocítica aguda infantil. HCPA [periódico na internet] 2010 [citado em out. 15]; 30(1):[cerca $\quad$ de $\quad 8 \quad$ p]. $\quad$ Disponível http://www.seer.ufrgs.br/index.php/hcpa/article/viewFile/11651/7510.

17.Brasil. Bulário Eletrônico da Angência Nacional de Vigilância Sanitária. Brasília (DF): Ministério da Saúde; 2008.

18.Pastore CA (editor). Diretrizes da Sociedade Brasileira de Cardiologia sobre análise e emissão de laudos eletrocardiográficos. Arq Bras Cardiol. 2009; 93(3):1-19.

19.Dahlof B, Devereux RB, Kjeldsen SE, Julius S, Beevers G, de Faire $U$ et al. Cardiovascular morbidity and mortality in the losartan intervention for endpoint reduction in hypertension study (LIFE): a randomized trial against atenolol. Lancet. 2002; 359:995-1003. 20.Aehlert B. Advanced Cardiac Life Support. 3 ed. Rio de Janeiro: Elsevier; 2007.

21.Pazin-Filho A, Santos JC, Castro RBP, Bueno CDF, Schmidt A. Parada Cardiorrespiratória. Medicina. 2003; 36:163-78.

22. Holmes CL, Patel BMP, Russell JA, Walley KR. Physiology of vasopressin relevant to management of septic shock. Chest. 2001; 120:989-1002. 


\section{Instrumento Para Coleta de Dados}

\begin{tabular}{|c|c|}
\hline \multicolumn{2}{|c|}{$\begin{array}{l}\text { - Preferencialmente, utilize caneta no preenchimento deste questionário } \\
\text { - Procure responder todas as questões } \\
\text { - Em caso de dúvida, entre em contato com o pesquisador para maiores } \\
\text { esclarecimentos. }\end{array}$} \\
\hline \multicolumn{2}{|c|}{ PARTE I - INFORMAÇÕES GERAIS } \\
\hline \multicolumn{2}{|l|}{ Identificação: } \\
\hline Unidade de Lotação: & Desde quando está lotado nesta instituição? \\
\hline $\begin{array}{l}\text { Qual o seu maior grau de Titulação? } \\
\begin{array}{ll}\text { ( ) Graduado } & \text { ( ) Especialista/Residência } \\
\text { ( ) Mestre } & \text { ( ) Doutor } \\
\text { ( ) Outro } & \end{array}\end{array}$ & 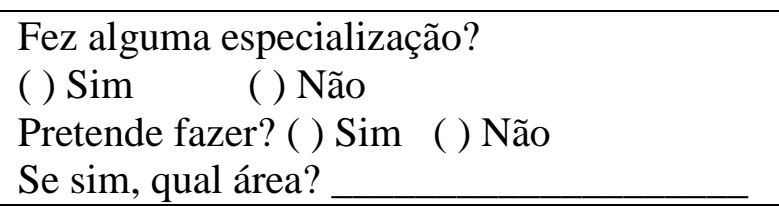 \\
\hline Tempo de Formado? & Tempo de Atuação em Emergência? \\
\hline \multicolumn{2}{|c|}{$\begin{array}{l}\text { Após a conclusão do seu curso de graduação em enfermagem, participou de alguma } \\
\text { atualização em farmacologia e administração de medicamentos? } \\
\begin{array}{lll}\text { ( ) Sim } & \text { ( ) Não } & \text { ( ) Não lembra }\end{array}\end{array}$} \\
\hline \multicolumn{2}{|c|}{$\begin{array}{l}\text { Como você definiria seu nível de conhecimento farmacológico das drogas usadas na sua } \\
\text { unidade de trabalho? } \\
\begin{array}{llll}\text { ( ) Ideal/Muito Satisfatório } & (\text { ) Bom/Satisfatório } & (\text { )Ruim/Insatisfatório }\end{array}\end{array}$} \\
\hline \multicolumn{2}{|c|}{$\begin{array}{l}\text { Existe em sua unidade um programa de educação permanente e/ou educação continuada que } \\
\text { contemple a temática Farmacologia e Administração de Medicamentos? } \\
\begin{array}{ll}\text { ( ) Sim } & \text { ( ) Não }\end{array}\end{array}$} \\
\hline \multicolumn{2}{|c|}{ 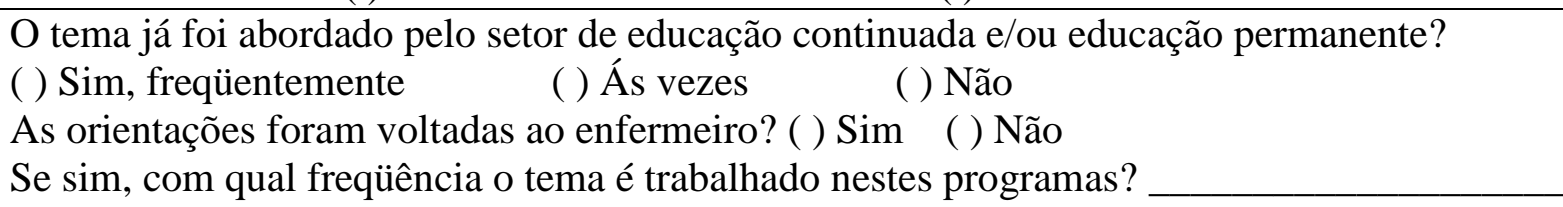 } \\
\hline $\begin{array}{l}\text { Existe na sua unidade algum protocolo } \\
\text { de medicamentos? ( ) Sim ( ) Não } \\
\text { Se sim, Houve treinamento para sua uti }\end{array}$ & anoro diluicõก odminictro \\
\hline
\end{tabular}




\begin{tabular}{|c|}
\hline PARTE II - CONHECIMENTOS SOBRE FARMACOLOGIA \\
\hline $\begin{array}{l}\text { As questões abaixo foram retiradas pré-teste do livro ACLS - Emergências em } \\
\text { Cardiologia (AEHLERT, 2007) e de coletâneas selecionadas para concursos na } \\
\text { área de Enfermagem }\end{array}$ \\
\hline $\begin{array}{l}\text { 01) Quais das seguintes drogas podem ser administradas por via endotraqueal quando } \\
\text { necessárias? } \\
\text { a) Amiodarona, dopamina, procainamida, naloxona e adenosina } \\
\text { b) Naloxona, atropina, epinefrina e lidocaína } \\
\text { c) Lidocaína, amiodarona, procainamida' e naloxona } \\
\text { d) Procainamida, epinefrina, lidocaína, adenosina e dopamina }\end{array}$ \\
\hline $\begin{array}{l}\text { 02) Os efeitos colaterais mais comuns da administração de amiodarona são: } \\
\text { a) Náuseas e assistolia } \\
\text { b) Bradicardia e hipotensão } \\
\text { c) Bloqueio AV e hipertensão } \\
\text { d) Visão turva e dor abdominal }\end{array}$ \\
\hline $\begin{array}{l}\text { 03) Nas doses recomendadas para uso em uma parada respiratória, a epinefrina e } \\
\text { vasopressina causam: } \\
\text { a) Causam potente vasoconstrição periférica } \\
\text { b) Neutralizam os ácidos acumulados durante a parada cardíaca } \\
\text { c) Retardam a condução através do nódulo atrioventricular } \\
\text { d) Produzem vasodilatação periférica }\end{array}$ \\
\hline $\begin{array}{l}\text { 04) Os inibidores da enzima conversora da angiotensina (ECA): } \\
\text { a) Aumentam a pressão arterial } \\
\text { b) Aumentam a carga de trabalho do coração } \\
\text { c) Podem ser utilizados no tratamento do infarto do miocárdio com elevação do } \\
\text { segmento ST } \\
\text { d) Incluem medicações como metoprolol, atenolol e propranolol }\end{array}$ \\
\hline $\begin{array}{l}\text { 05) A lidocaína pode ser LETAL quando administrada em qual dos ritmos a seguir? } \\
\text { a) Taquicardia ventricular monomórfica } \\
\text { b) Ritmo Idioventricular (Escape Ventricular) } \\
\text { c) Taquicardia Ventricular polimórfica } \\
\text { d) Taquicardia Sinusal }\end{array}$ \\
\hline $\begin{array}{l}\text { 06) Várias medicações são utilizadas na parada cardiorespiratória (PCR). Dentre eles, } \\
\text { estão: atropina e bicarbonato de sódio. A utilização de bicarbonato de sódio tem o } \\
\text { intuito de: } \\
\text { a) Aumentar a força contrátil cardíaca } \\
\text { b) Aumentar o tônus muscular miocárdico } \\
\text { c) Corrigir a hipoxemia } \\
\text { d) Corrigir a acidose metabólica }\end{array}$ \\
\hline
\end{tabular}


07) Durante o atendimento a uma parada cardiopulmonar deve-se estar atento ao tempo de intervalo entre as doses de atropina. Qual o intervalo ideal entre as doses da atropina?

a) 3 a 5 minutos

b) 4 a 6 minutos

c) De até 3 minutos

d) De até 6 minutos

08) Cliente da emergência que recebe doses de morfina deve ser monitorado quanto a?

a) Freqüência respiratória, pressão arterial, pulso e nível de consciência

b) Temperatura, nível de consciência, pressão arterial e respiração

c) Diurese, temperatura, pressão arterial e nível de consciência

d) Nível de consciência, respiração, temperatura e diurese

09) Um cliente deu entrada na unidade de emergência com quadro de crise convulsiva. Qual a medicação indicada para cessar a crise convulsiva, caso esta esteja prolongada?
a) Amiodarona
b) Diazepan
c) Efedrina
d) Manitol 